\section{Determinantes sociales de la malaria... ¿Aportes de la Medicina Social Latinoamericana?}

Apreciados editores:

Después de la lectura del ensayo "Malaria y determinantes sociales de la salud: un nuevo marco heurístico desde la medicina social latinoamericana" (1), consideramos pertinente discutir los potenciales nuevos aportes de esta propuesta. El autor parte afirmando que la investigación de la malaria ha seguido predominantemente el paradigma positivista y la concepción biomédica de la enfermedad, y sugiere que este enfoque es incompleto y que poco ha contribuido al mejoramiento de las condiciones de vida de la población y a la disminución de su carga epidemiológica. Además, argumenta, con ímpetu de novedad, que la malaria debería entenderse como un proceso biológico y social, y que ello puede hacerse mediante la medicina social latinoamericana (1).

Afirmaciones similares fueron hechas en la década de los 70, cuando la medicina social latinoamericana era una disciplina emergente, y están basadas en conceptos de la economía política, que postulan que la organización social y las estructuras del poder económico son determinantes fundamentales de la salud, lo cual hoy es generalmente aceptado. Sin embargo, aseverar que este marco debe usarse para entender la malaria en la segunda década del siglo XXI es debatible. Hacerlo es desdeñar los trabajos seminales sobre determinantes sociales y su abordaje por la epidemiología social de Yankauer y Syme -por sólo citar unos ejemplospublicados desde la década de los $50(2,3)$, y específicamente el escrito de Banguero (4), quien en 1984 ya había esbozado un modelo explicativo de la malaria en Colombia, basado en factores sociales, económicos y biológicos.

Algunos trabajos, sin seguir los postulados de la medicina social latinoamericana, fueron publicados posteriormente reportando con evidencia propia que estos determinantes sociales son relevantes en la presentación de la malaria e, incluso, sugiriendo que se trataría de una relación de doble vía, puesto que esta enfermedad parece, a su

Correspondencia:

Julián Alfredo Fernández

jfernandeznino@yahoo.com vez, ser un determinante de la persistencia de la pobreza en los países endémicos (5). Por otro lado, la disminución de la presentación de la malaria en muchos países se debió, probablemente en gran medida, a cambios en estos determinantes económicos (6) pero sin recurrir a la medicina social latinoamericana como referente, sino más bien, al mejoramiento general del nivel de vida, el saneamiento ambiental y el uso de lo que en esa época se llamó "medicina social" y que, según algunas tesis, derivó en lo que ahora conocemos como epidemiología (7). Por lo anterior, este ensayo parece más una apología de la medicina social latinoamericana que la presentación de un modelo explicativo novedoso.

Este trabajo parece apelar a la necesidad de un marco altamente comprehensivo de la malaria. Sin embargo, debe considerarse que, en general, las diversas disciplinas científicas no pretenden ser altamente comprehensivas cada investigador hace sus aportes desde y dentro de sus propios paradigmas. Unacomprensiónholísticacorresponde más a la visión que podría y debería obtener, por ejemplo, un salubrista a partir del conocimiento de las diversas disciplinas, para así determinar las mejores formas de intervenir mediante la integración del conocimiento transdisciplinario en teorías amplias, más no necesariamente "totalizantes" como pareciera ser la naturaleza de esta propuesta. En este sentido, este trabajo agrupa conocimientos de diversas disciplinas y las organiza, pero no muestra claramente como llevar a la praxis su aproximación "heurística". De hecho, en salud pública se ha debatido que muchos modelos teóricos tienden a centrarse, como podría ser éste el caso, en su valor metafórico o representativo más que en su desarrollo teórico, y en el caso de la medicina social latinoamericana esto se ha hecho sobredimensionando la representación de "lo social" (8), dejando de lado la evaluación permanente de los postulados teóricos, necesariamente mediante la investigación empírica y la práctica disciplinaria (9) y no solamente mediante el ejercicio de la dialéctica.

En esta propuesta la organización estructural de los constructos y sus relaciones dentro de un marco conceptual puede ser novedosa, pero el modelo explicativo -y la teoría general que lo contienesolamente retoma los viejos postulados de la 
medicina social latinoamericana, sin que sea visible la manera en que, como teoría científica, debería servir específicamente en malaria para además de "diagnosticar un fenómeno y explicar su proceso"como podría hacerlo- contribuir también a "predecir desenlaces específicos" mediante la generación y falsificación de nuevas hipótesis (10) que permitan identificar, por ejemplo, nuevos determinantes relevantes que intervengan la infección o evaluar las mejores estrategias de intervención para la prevención y el control de la malaria.

En contraste, el enfoque clásico empírico-positivista puede no ser tan comprehensivo y holístico, pero su praxis ha sido históricamente evidente, como en la identificación de la eficacia de los toldillos impregnados con insecticida en la reducción de la mortalidad por malaria en regiones puntuales (11), impactos potencialmente extensibles si se implementaran más amplia y eficientemente estas evidencias en las naciones endémicas. Lo anterior no quiere decir que esta aproximación no sea perfectible y que amerite complementarse e incluso superarse como teoría y modelo del fenómeno, sino que, intrínsecamente, es eficiente y que además debe evaluarse considerando que el éxito de un paradigma en el control de la enfermedad no depende solamente de la validez de las teorías que la estudian, sino de la existencia de voluntad política, factibilidad técnica, contexto social y disponibilidad de recursos.

No se discute aquí, por ejemplo, el efecto potencial de la reducción de la pobreza sobre la malaria (6), sino que se controvierte que estos cambios deban plantearse privilegiadamente desde la perspectiva de la medicina social latinoamericana. Los marcos propuestos por la teoría eco-social podrían ser un camino novedoso de entendimiento e intervención más inmediato y factible sobre la malaria que, incluso, retoma elementos teóricos de la medicina social integrando elementos del análisis a múltiples niveles, en modelos que pueden someterse a la evaluación empírica (12).

Consideramos que aún sigue siendo un gran reto la formulación de mejores y nuevos modelos conceptuales deladeterminación socialdela malaria, pero el desafío quizás más importante es evaluar y aplicar paradigmas de investigación/intervención que generen, no sólo mayor conocimiento, sino que también impulsen la mejoría de salud de las poblaciones afectadas históricamente por esta enfermedad y cuya reivindicación social es necesaria e inaplazable.

Julián Alfredo Fernández, Grupo de Infecciones y Salud en el Trópico, Universidad Nacional de Colombia, Bogotá, D.C., Colombia

Alvaro Javier Idrovo, Centro de Investigación en Sistemas de Salud, Instituto Nacional de Salud Pública, Cuernavaca, Morelos, México

Carlos Andrés Castañeda, Facultad de Economía, Universidad Nacional de Colombia, Bogotá, D.C., Colombia

Vanesa Giraldo-Gärtner, Facultad de Ciencias Jurídicas y Sociales, Universidad de Caldas, Manizales, Colombia

\section{Referencias}

1. Piñeros JG. Malaria y determinantes sociales de la salud: un nuevo marco heurístico desde la medicina social latinoamericana. Biomédica. 2010;29 (en prensa).

2. Yankauer A. The relation of fetal and infant mortality to residential segregation. An inquiry of social epidemiology. Am Sociol Rev. 1950;15:644-8.

3. Syme SL. Social determinants of disease. Ann Clin Res.1987;19:44-52.

4. Banguero H. Socioeconomic factors associated with malaria in Colombia. Soc Sci Med. 1984;19:1099-104.

5. Sachs J, Malaney P. The economical and social burden of malaria. Nature. 2002;415:680-5.

6. Straton L, O'Neill MS, Kruk M, Bell ML. The persistent problem of malaria: Addressing the fundamental causes of a global killer. Soc Sci Med. 2008;67:854-62.

7. Gunning-Schepers LJ. That which we call social medicine... J Epidemiol Community Health. 1997;51:224-6.

8. Arredondo-López A. Modelos y conceptos en salud pública y sistemas de salud: hacia una perspectiva transdisciplinaria. Cuad Méd Soc (Chile). 2009;49:26-35.

9. Kaplan N. What's wrong with social epidemiology, and how can we make it better? Epidemiol Rev. 2004;26:124-35.

10. Ostrom E. Institutional rational choice: an assessment of the institutional analysis and development framework. En: Sabatier PA. Theories of the policie process. Boulder, CO: Westview Press; 1999. p. 35-71.

11. Choi HW, Breman JG, Teutsch SM, Liu S, Hightower AW, Sexton TD. The effectiveness of insecticide-impregnated bed nets in reducing cases of malaria infection: a meta-analysis of published results. Am J Trop Med Hyg.1995;52:377-82.

12. Krieger $\mathbf{N}$. Theories for social epidemiology in the 21st century: an ecosocial perspective. Int $\mathrm{J}$ Epidemiol. $2001 ; 30: 668-77$ 


\section{Respuesta carta al editor}

Señores

Comité editorial

Revista Biomédica

Este salubrista en formación agradece enormemente las contribuciones de sus lectores, no obstante, debo discernir de algunas de ellas.

Primero, quisiera aclarar que el "ímpetu de novedad" con que los lectores calificaron mis argumentos frente a la forma en que propongo debería entenderse la malaria, corresponde a una equivocada interpretación del texto, la cual también se presenta cuando me adjudican una supuesta apología de la medicina social latinoamericana.

Como bien señalan, la concepción de saludenfermedad del ensayo corresponde a la propuesta por el movimiento de medicina social latinoamericana. Este enfoque se asumió debido a que, al permitir una construcción más compleja de la realidad de salud en relación con la realidad socio-histórica presente en un lugar y momento concreto, el conocimiento generado puede ser útil como herramienta política (1). De esta forma, trasciende su uso habitual como insumo para diseñar estrategias técnicas puntuales, a las cuales también puede contribuir. Esto no significa necesariamente marcos altamente comprehensivos. Significa capacidad de dialogo interdisciplinario y, eso sí, de diálogo intercultural en la construcción del conocimiento, un reto grande que requiere apertura y sobre todo creatividad (1).

Comparto con ellos la afirmación sobre el mayor énfasis de la medicina social latinoamericana en la crítica teórica y su escaso ejercicio empírico. Debe aclararse que esta situación no es caprichosa y la principal razón para que esto ocurriera es que, en las tres últimas décadas del siglo XX, los sistemas de ciencia y tecnología de los países de la región privilegiaron un discurso hegemónico de la ciencia y miraron con sospecha aquellas propuesta basadas en concepciones alternativas, sobre todo las construidas desde el materialismo histórico (2).

Una mirada así de amplia es fundamental para buscar la solución del problema de la malaria desde sus causas "profundas", desde sus determinantes, la inequidad y la pobreza. Entender precisamente estos fenómenos y los mecanismos socio-históricos que los generan es prioritario para comprender procesos de salud-enfermedad como la malaria y generar estrategias políticas a una escala mayor que la intervención biomédica (3).

En Colombia, cuatro estudios han contemplado la malaria desde los determinantes sociales de la salud y todos señalan que el control de esta enfermedad debe partir de transformaciones más amplias que acompañen intervenciones específicas (4-7). Este ensayo plantea lo mismo e intenta instrumentalizar de alguna forma la realidad socio-histórica y su carácter dinámico, con el objetivo de que su estudio permita formular soluciones más amplias, pero a la vez concretas.

Juan Gabriel Piñeros, Grupo de Malaria, Universidad de Antioquia, Medellín, Colombia

\section{Referencias}

1. Breilh J. Epidemiología crítica. Ciencia emancipadora e interculturalidad. Buenos Aires, Argentina: Lugar Editorial; 2003.

2. Briceño-León R. Endemias, epidemias y modas: la sociología de la salud en América Latina. Revista Española de Sociología. 2003;3:69-85

3. Breilh J, Tillería Y. Aceleración global y despojo en Ecuador. El retroceso del derecho a la salud en la era neoliberal. Quito, Ecuador: Universidad Andina Simón Bolívar/AbyaYala; 2009

4. Bonilla-Castro E, Kuratomi LS, Rodríguez P, Rodríguez A. Salud y desarrollo. Aspectos socioeconómicos de la malaria en Colombia. Bogotá, Colombia: Plaza y Janes Editores; 1991.

5. Franco-Agudelo S. El paludismo en América Latina. Guadalajara, México: Editorial Universidad de Guadalajara; 1990.

6. Sevilla-Casas E. Human mobility and malaria risk in the Naya river basin of Colombia. Soc Sci Med. 1993;37:115567.

7. Valero-Bernal MV. Persistencia, resurgimiento y resistencia de la malaria en Colombia: 1960-2004 teniendo en cuenta las dimensiones socio-políticas, culturales y del comportamiento individual y colectivo y sus implicaciones en las políticas de control. Informe final, doctorado interfacultades en Salud Pública. Bogotá: Universidad Nacional de Colombia; 2007. 